\section{Should Pharmacy Informatics Officer Positions be Based in, and Report to, the Pharmacy Department, Rather than the Health Information Technology Department?}

\section{THE "PRO" SIDE}

Pharmacy computer systems have been around since the 1970s but were initially used primarily in the retail pharmacy sector. It was not until the late 1980s and early 1990s that computerized pharmacy information systems began to become common in hospital pharmacy departments. The primary objective of computerizing hospital pharmacies in those early years was for inventory management and financial reporting. However, in the past decade, the situation has changed quite dramatically.

In 1999, when the Institute of Medicine published To Err Is Human: Building a Safer Health System, ${ }^{1}$ the human and financial costs of medical error could no longer be ignored. The Institute of Medicine report revealed that medical errors occur routinely in the hospital setting and that tens of thousands of patients die in the United States as a result of medical errors each year. The report also indicated that medication errors represent the largest single cause of medical errors in the hospital setting. In 2004, the Canadian Adverse Events Study suggested that 9000 to 23000 Canadians die each year from adverse events that could have been prevented. ${ }^{2}$ These reports highlighted the need to change the way in which health care was delivered and the need for better systems to improve patient safety. In 2003, an analysis by the US-based Leapfrog Group indicated that computerized prescriber order entry (CPOE) systems could reduce serious medication errors by $55 \%$. $^{3}$ This was the turning point for many hospitals and the start of a new era in the utilization of computerized systems to reduce the error rate associated with the ordering and dispensing of medications.

Many Canadian hospitals embarked on a journey toward CPOE systems and other computerized systems that use clinical decision-support software. As might be expected with any major change like this one, new challenges arose. Many hospitals initially did not factor in the need for clinical input to the building and maintenance of these systems. It soon became obvious that computerized systems alone could not improve patient care and safety. Both a technical understanding of the information technology and the appropriate clinical knowledge are required to realize the full promise of these systems.

Integrating a pharmacy clinical informatics team into the pharmacy department is a key requirement for successful implementation and maintenance of computerized pharmacy systems. Having staff members in the pharmacy department who have an intimate knowledge of how both the department and the technology operate is critical to the safe and effective use of these systems. The pharmacy's clinical informatics staff members need to maintain day-to-day contact with the pharmacy's other staff members to ensure that the link between the technical and clinical aspects of the systems are optimized, from the starting point of purchasing and receiving the medication to the point at which the medication is given to the patient.

In the current hospital pharmacy environment of CPOE, automated dispensing machines, smart IV pumps, robotic admixture, electronic medication administration records, and bar-coded medication administration systems, there is no single integrated system that is capable of fulfilling all the necessary requirements for automation and documentation. As such, integration of various systems is required to ensure safe prescribing, distribution, and administration of medications. Ongoing monitoring of interface logs, coordinated testing of change initiatives, implementation of upgrades, and continual trouble-shooting are all activities that must be undertaken by a pharmacy clinical informatics team. Medication distribution is not static, and changes in practice and protocols occur almost daily. New products are added to the systems because of changes in clinical needs, drug shortages, or formulary changes. New clinical guidelines, restrictions, or treatment algorithms are implemented and must be incorporated into the medication ordering and dispensing processes. When such changes occur, the clinical informatics staff members must be available to work with the clinical or operational pharmacy staff to implement any necessary changes in the computerized systems.

Having the pharmacy clinical informatics team as part of the pharmacy department also ensures a collaborative environment for trouble-shooting and looking for innovative solutions to technical or system problems. Pharmacy staff members feel more comfortable in trying new systems or processes when they know that they are supported by a dedicated pharmacy informatics team, with members who understand the need for safe medication systems, as well as the technological needs.

When implementing new medication ordering processes, order sets, or new technology, it is invaluable for the informatics personnel to be able to work directly with the clinical pharmacists involved with the team or program that is implementing the change. Being part of the pharmacy department provides flexibility to the clinical informatics team and allows for the secondment of clinical pharmacists for set periods of time to work on the development and implementation of special projects. This ease of sharing resources provides the pharmacy clinical informatics team with the clinical input needed to develop a system that works for the end-user without compromising patient safety. 
With the increased demand for data mining and reporting for research and for operational and clinical activities, it is essential to have the ability within the pharmacy department to access the information available in the pharmacy systems. Having the clinical informatics staff work with the clinical and operational pharmacy staff to analyze and validate data for various reports ensures the integrity of the data. It also ensures that pharmacy staff can generate and customize any required reports.

When information systems are running smoothly, all is well. However, all technology-based systems go down at some point, either on a planned or an unplanned basis. At these times, it is invaluable to have the pharmacy clinical informatics team available to immediately address the contingency issues, such as down-time procedures, and to provide the link between the pharmacy department and any external technical teams whose input may be required.

The arguments advanced here support the need for a pharmacy clinical informatics group based in the pharmacy department. If there is a designated leader for the group, with a title such as chief pharmacy information officer, it would seem only reasonable that this individual should also be based in, and report through, the pharmacy department. Although it is important to have a strong linkage with the e-health department, it would be problematic from an accountability and chain-of-command perspective to have staff working in pharmacy but reporting to another department.

\section{References}

1. Kohn LT, Corrigan JM, Donaldson MS, editors. To err is human: building a safer health system. Washington (DC): National Academy Press, Institute of Medicine; 2000 [cited 2011 Aug]. Available from: www.nap.edu/ catalog.php? record_id=9728\#toc

2. Baker GR, Norton PG, Flintoft V, Blais R, Brown A, Cox J, et al. The Canadian Adverse Events Study: the incidence of adverse events among hospital patients in Canada. CMAJ 2004;170(11):1678-1686.

3. Birkmeyer JD, Dimick JB. The Leapfrog Group's patient safety practices, 2003: the potential benefits of universal adoption. Washington (DC): Leapfrog Group; 2004 [cited 2011 Aug]. Available from: www. leapfroggroup.org/media/file/Leapfrog-Birkmeyer.pdf

Monique Pitre, RPh, BScPhm, FCSHP

Manager, Pharmacy Clinical Informatics

University Health Network

Toronto, Ontario

\section{THE "CON" SIDE}

Pharmacy informatics officer positions are the product of the information technology wave that is sweeping through health care. Until very recently, the importance of these positions to pharmacy and to the overall health care system was not well conceptualized or well understood. Regional, provincial, and pan-Canadian initiatives to share health information across the continuum of patient care have highlighted the need for such positions at all levels of planning and implementation of health information technology.
Pharmacy has historically been an early adopter of technology and automation, particularly when such technologies have supported drug distribution systems. ${ }^{1}$ Pharmacy departments in the hospital environment recognized decades ago that technology could play an important role in increasing the efficiency, effectiveness, and safety of medication management processes. ${ }^{2}$ In turn, hospital pharmacy managers and staff played key roles in defining the requirements for pharmacy information systems, evaluating available software systems, and selecting and implementing the preferred system. After implementation, management of the pharmacy information system, as well as automated repackaging and dispensing technologies, largely resided within the pharmacy department. Intersection with other clinical systems was minimal. This departmental ownership of the pharmacy information system and automated drug distribution systems suited needs at that time. Health information technology departments were utilized for technical support, providing consultative services on technical requirements during the selection process, facilitating the acquisition and set-up of hardware and software, and providing ongoing technical support for departmental systems.

As a result of regionalization initiatives during the 1990s, organizational structures changed in both pharmacy and health information technology. A broader regional strategic vision evolved. Pharmacy informatics positions were created at the regional level, and these positions generally, but not always, reported through the regional pharmacy organizational structure. The need for these positions continues, both to lead regional pharmacy informatics initiatives and to ensure that regional standards and practices are maintained.

In recent years, jurisdictional boundaries have changed yet again. Most provinces have established health information technology organizations with a provincial mandate. These organizations have been charged with facilitating the transformation of health care delivery, usually at a provincial level, through the use of information technologies. The vision and strategic plan of each provincial health information technology organization reflect its province-wide mandate, as well as health information technology needs across the continuum of patient care. The reframing of the health information technology concept as "e-health" reflects this broadening perspective.

Given the momentum of integration of health information technology, clinical leaders such as chief medical information officers have been brought into e-health organizations to bridge the gap between technical personnel and clinicians. Leaders in health information technology understand that information systems to support medication therapy management are critical for achieving optimal patient outcomes, ${ }^{2}$ and they recognize the need for pharmacists to be part of senior health information technology teams. ${ }^{3}$ To address this need, progressive organizations have created the position of chief pharmacy informatics officer. Those appointed to such positions bring a clinical background, pharmacy business knowledge, and a vision for optimal medication therapy management to the e-health senior management group.

The chief pharmacy informatics officer is responsible for promoting seamless flow of medication-related information through all transitions of care, not just acute care. The Canada 
Health Infoway initiative, which promotes applications for electronic medical records in primary care, is but one example of the opportunities for enhanced flow of medication-related information. E-prescriptions, submitted from electronic medical records to a provincial pharmacy information system, are now used by retail pharmacists for dispensing and by hospital pharmacists for accurate medication histories. Unfortunately, poor or nonexistent communication of medication information at transition points (admission, transfer, discharge) is responsible for as many as $50 \%$ of all medication errors and up to $20 \%$ of all adverse drug events in hospitals. ${ }^{4}$ Access to a provincial pharmacy information system with active e-prescription information represents a tremendous opportunity to address the present shortfalls in medication information flow.

Positioning of the chief pharmacy informatics officer within the provincial e-health senior management structure does not preclude the presence of other pharmacy informatics officer positions within hospital, long-term care, or community pharmacy structures. In fact, a collaborative relationship between the chief pharmacy informatics officer and other pharmacy informatics officers within every pharmacy organizational structure is essential to ensure that the needs and perspectives of all areas of pharmacy practice are integrated into the overall provincial e-health strategy. In addition, pharmacy informatics officers within various practice areas are needed to advocate for the needs of their respective practices.

The Blueprint for Pharmacy addresses the intersections of pharmacy practice and health information technology systems. ${ }^{5}$ It advocates for visible involvement of pharmacists at the levels where decisions are made about integrated clinical information solutions, particularly those that affect medication management across the continuum of patient care. A chief pharmacy informatics officer, positioned as a member of the e-health senior management group, would fulfill that role. Specific actions that would fall within this person's responsibilities include the following:

- Engage the pharmacy profession in the development and implementation of health information technology initiatives across Canada so that the interests of both pharmacists and patients are represented.

- $\quad$ Provide input into the development and implementation of secure health information technology systems in hospital, community, primary care, and long-term care settings.
- Advocate for effective health information technology communication strategies that provide timely and relevant information to the pharmacy community.

- Support the role of pharmacists in medication management, by influencing, developing, and implementing policies and practices relevant to electronic transfer of prescriptions, electronic signatures, and electronic prescribing.

It is no longer in pharmacy's best interest for all pharmacy informatics officer positions to be based within, and report through, pharmacy organizational structures. Chief pharmacy informatics officer positions, and certain other related positions, will be most effective when they are part of the e-health organizational structure and are able to advocate at the appropriate e-health jurisdictional level. Working in direct collaboration with the chief medical informatics officer and other e-health clinical leaders, these people will be better positioned to help establish an infrastructure that not only supports day-to-day pharmacy operations, but also provides medication information and other technology support to caregivers in an accurate and timely manner. In the end, the best approach is one that focuses on patient care across the continuum of care.

\section{References}

1. Siska MH, Tribble DA. Opportunities and challenges related to technology in supporting optimal pharmacy practice models in hospitals and health systems. Am J Health Syst Pharm 2011;68(12):1116-1126.

2. Fox BI, Poikonen J, Gumpper K. Sharing experiences with information technology. Am J Health Syst Pharm 2008;65(11):1012, 1014.

3. Hagland M. Is there a pharmacist in the house? When it comes to clinical IT implementations, healthcare IT leaders are turning to the "third discipline" for leadership and involvement in clinical informatics. Healthc Inform 2010;27(12):18-22.

4. Reconcile medications at all transition points. Cambridge (MA): Institute for Healthcare Improvement; [cited 2011 Jul 19]. Available from: www.ihi.org/ knowledge/Pages/Changes/ReconcileMedicationsatAllTransitionPoints.aspx

5. Task Force on a Blueprint for Pharmacy. Blueprint for pharmacy: implementation plan. Ottawa (ON): Canadian Pharmacists Association; 2009 Sep [cited 2011 Jul 19]. Available from: www.pharmacists.ca/content/ about_cpha/whats_happening/cpha_in_action/pdf/BlueprintImplementation Plan.pdf

Natalie Thickson, BscPharm, CPHIMS-CA

Clinical Technology

Manitoba e-Health

Winnipeg, Manitoba 